\title{
Differential rotation decay in the radiative envelopes of CP stars
}

\author{
R. Arlt ${ }^{1}$, R. Hollerbach ${ }^{2}$, and G. Rüdiger ${ }^{1}$ \\ 1 Astrophysikalisches Institut Potsdam, An der Sternwarte 16, 14482 Potsdam, Germany \\ 2 Department of Mathematics, University of Glasgow, Glasgow G12 8QW, UK
}

Received 2 July 2002 / Accepted 4 February 2003

\begin{abstract}
Stars of spectral classes A and late B are almost entirely radiative. CP stars are a slowly rotating subgroup of these stars. It is possible that they possessed long-lived accretion disks in their T Tauri phase. Magnetic coupling of disk and star leads to rotational braking at the surface of the star. Microscopic viscosities are extremely small and will not be able to reduce the rotation rate of the core of the star. We investigate the question of whether the magneto-rotational instability can provide turbulent angular momentum transport. We discuss whether differential rotation is present in CP stars. Numerical MHD simulations of thick stellar shells are performed. An initial differential rotation law is subject to the influence of a magnetic field. This configuration does indeed give rise to a magneto-rotational instability. The emerging flows and magnetic fields efficiently transport angular momentum outwards. Weak dependence on the magnetic Prandtl number $\left(\sim 10^{-2}\right.$ in stars $)$ is found from the simulations. First tests with stratified shells show different flows, but the same efficiency of angular-momentum transport. Since the estimated time-scale of decay of differential rotation is $10^{7}-10^{8} \mathrm{yr}$, and comparable to the life-time of A stars, we find the braking of the core to be an ongoing process in many CP stars. The evolution of the surface rotation of CP stars with age will be an observational challenge, and of considerable value in verifying these simulations.
\end{abstract}

Key words. stars: chemically peculiar - stars: rotation - stars: magnetic fields - magnetohydrodynamics (MHD) - turbulence

\section{Introduction}

The present work was originally motivated by the existence of stars of spectral type A, of which a subgroup is distinguished by their strong magnetic fields. These peculiar A and late B stars, which are collectively called CP stars, rotate significantly slower than their non-magnetic relatives. The dipole axes of the magnetic fields of CP stars have various orientations, with a tendency to larger tilts at faster rotation (Landstreet \& Mathys 2000).

Young stars suffer from considerable rotational braking, in particular during their pre-main sequence evolutionary phase. Angular momentum is lost partly through stellar winds in the $\mathrm{T}$ Tauri phase. Additional braking probably applies to T Tauri stars having an accretion disk (classical T Tauri stars - CTTS). These stars also form a slowly rotating subgroup among the T Tauri stars (Bouvier et al. 1993), just as the slow CP stars do among ordinary A and late B stars. Magnetic fields exiting the accretion disk will couple to the stellar field and exert torques as well. The braking is efficient for solar-mass T Tauri stars, as found by Cameron \& Campbell (1993). Recently, Stępien (2000) proposed the analogy between the CP star subgroup and the CTTS among T Tauri stars and explains the slow $\mathrm{CP}$ stars by rotational locking with the disk in their pre-mainsequence phase.

If stars are considerably slowed down at the surface, how fast do their interiors rotate? The microscopic viscosity of

Send offprint requests to: $\mathrm{R}$. Arlt, e-mail: rarlt@aip.de stellar plasma is extremely small. We can estimate the timescale of viscous decrease of the rotation in case of external braking simply by estimating the time-scale due to the gas's viscosity of roughly $v=10 \mathrm{~cm}^{2} / \mathrm{s}$. For a stellar radius of $R=10^{11} \mathrm{~cm}$, we get a viscous time-scale of $R^{2} / v \approx 10^{13} \mathrm{yr}$ which is $4-5$ orders of magnitudes longer than the life-time of A stars and even a thousand times more than the age of the universe. Viscous decay of the differential rotation in any radiative shell of a star is thus not applicable. As long as there is no convection providing turbulent transport of angular momentum (whose efficiency may, however, not be very high), we should expect strong differential rotation on the way from the stellar surface to the deep interior.

Differential rotation in a radiative star will be prone to the magneto-rotational instability, which requires only two things: an angular velocity decreasing with axis distance and a weak magnetic field (Balbus \& Hawley 1991). The question of what "weak" means will be addressed in Section 2 in the discussion of the initial magnetic field used for our simulations. The instability is known to evolve quickly on the time-scale of the rotation period. It has turned out to be an efficient generator of turbulence in accretion disks. See e.g. Kitchatinov \& Rüdiger (1997) for a linear global analysis and Hawley (2000) and Arlt \& Rüdiger (2001) for simulations. However, the instability is equally applicable to a stellar interior as long as the angular velocity decreases with axis distance in parts of the spherical domain. The mechanism is typically termed magneto-rotational instability or Balbus-Hawley instability. It is a consequence of 
the local and linear MHD equations; a lower limit to the magnetic field is only imposed by the magnetic diffusivity $\eta$ which is extremely small for stellar plasma. Field geometry is also almost irrelevant for the onset of the instability. The magnetorotational instability must be quite ubiquitous in stellar radiative zones as soon as differential rotation emerges, likely to be caused by surface braking.

The effect of magneto-rotational instability in radiative stellar zones was approached earlier by Balbus \& Hawley (1994) with a local, linear analysis. Their result is that the instability does not lead to radial transport of angular momentum because of the stabilizing radial stratification. Transport would emerge on spherical shells and deposit angular momentum in the equatorial plane.

We would like to address the question of how long it takes to turn the radiative envelope of the star into a uniformly rotating shell. Is there any chance to maintain a rotation profile depending only on the axis distance? The answer of whether or not differential rotation may be expected will have consequences for the geometry of magnetic fields or possibly for their generation in CP stars.

The questions were investigated numerically. The simulations described in Sect. 2 are fully global and non-linear, but do not possess density gradients or buoyancy. This makes the configuration different from the linear analysis by Balbus \& Hawley (1994) which was only linear, but included buoyancy. While the full global model has not yet been accessible, we also present first results from density-stratified calculations. The third to fifth sections deal with the hydrodynamic and magnetohydrodynamic evolution of a thick spherical shell. We will discuss the consequences of the computations in Sect. 6.

\section{Simulations}

The simulations apply the spectral, spherical MHD code of Hollerbach (2000). The computational domain covers a full spherical shell from the inner radius $r_{\mathrm{i}}=0.2$ to the outer radius $r_{\mathrm{o}}=1.0$. We start from the non-ideal MHD equations with the kinematic viscosity $v$ and the magnetic diffusivity $\eta$. The time-dependent, incompressible, non-dimensional equations are then

$$
\begin{aligned}
& \left(\frac{\partial}{\partial t}+\boldsymbol{u} \cdot \nabla\right) \boldsymbol{u}=-\nabla p+P m \nabla^{2} \boldsymbol{u}+(\nabla \times \boldsymbol{B}) \times \boldsymbol{B} \\
& \frac{\partial \boldsymbol{B}}{\partial t}=\nabla^{2} \boldsymbol{B}+\nabla \times(\boldsymbol{u} \times \boldsymbol{B})
\end{aligned}
$$

with the usual meanings of $\boldsymbol{u}, \boldsymbol{B}$, and $p$ as the velocity, magnetic field, and pressure which is not explicitly calculated in this model, but eliminated by applying the curl-operator to Eq. (1). We integrate the equations in a non-rotating frame of reference. The rotation is thus entirely made up of $\boldsymbol{u}$.

Lengths are normalized with the radius of the sphere, $R$, times are measured in diffusion times $R^{2} / \eta$, velocities are normalized with $\eta / R$ as well as magnetic fields with $\sqrt{\mu \rho} \eta / R$. Note that the permeability $\mu$ and the density $\rho$ are constants in space and time in our approach. This normalization leads to the magnetic Prandtl number,

$P m=\frac{v}{\eta}$

measuring the ratio of diffusive to viscous time-scales.

Instead of the physical $\boldsymbol{u}$ and $\boldsymbol{B}$, we integrate the potentials $e, f, g$, and $h$ which compose the physical quantities by

$\boldsymbol{u}=\nabla \times(e \hat{\boldsymbol{r}})+\nabla \times \nabla \times(f \hat{\boldsymbol{r}})$,

$\boldsymbol{B}=\nabla \times(g \hat{\boldsymbol{r}})+\nabla \times \nabla \times(h \hat{\boldsymbol{r}})$,

where $\hat{\boldsymbol{r}}$ is the unit vector in radial direction. The potentials for $\boldsymbol{u}$ and $\boldsymbol{B}$ are decomposed into Chebyshev polynomials for the radial dependence and into spherical harmonics for the angular dependence. The representation by potentials implies that $\nabla$. $\boldsymbol{u}=0$ and $\nabla \cdot \boldsymbol{B}=0$ are always fulfilled automatically.

The initial conditions for the velocity represents a rotation profile in which the angular velocity decreases with the cylinder radius, $s=r \sin \theta$, according to

$\Omega=\frac{R m}{\sqrt{1+(2 s)^{2 q}}}$,

where $R m$ is the magnetic Reynolds number, which is determined by the normalized angular velocity on the axis,

$R m=\frac{R^{2} \Omega_{0}}{\eta}$.

This $R m$ will be varied in our simulations; we always put $q=2$. A profile depending on the axis distance appears to be a reasonable choice for the internal rotation of a star being prone to magnetic coupling with an accretion disk.

According to the Rayleigh criterion of hydrodynamic stability,

$\frac{\mathrm{d} j^{2}}{\mathrm{~d} s}>0$

where $j=s^{2} \Omega$ is the angular momentum per unit mass, the above rotation profile (6) will provide us with a hydrodynamically stable configuration. For large axis distances $s \gg 1$ the profile with $q=2$ would be marginally stable, but within our shell of finite radius, the Rayleigh criterion is not violated.

The construction of the initial magnetic field is based on a vertical, homogeneous field, onto which we impose a nonaxisymmetric perturbation of Fourier mode $m=1$. The total initial magnetic field can be written as

$\boldsymbol{B}=B_{0}[\hat{z}+\epsilon \sin (k z+\pi / 4) \hat{\boldsymbol{x}}]$,

where $\hat{z}$ is the unit vector in the direction of the rotation axis and $\hat{\boldsymbol{x}}$ is a unit vector in the equatorial plane. The wave number of the perturbation is $k=4 \pi$. We added $\pi / 4$ to the second term in (9) in order to provide mixed parity to the system. Equatorial and axial symmetry are thus broken allowing the system to develop flows and fields in all modes.

The initial magnetic field contains $B_{0}=100$ and $\epsilon=1$ in most of our simulations. At $R m=10000$, this configuration implies a magnetic energy which is two orders of magnitude smaller than the kinetic energy in the initial rotation (precisely $E_{\text {mag }}=0.017 E_{\text {kin }}$ ). The magnetic energy is thus much 
smaller than the rotational energy as required for the onset of the magneto-rotational instability. In a real stellar environment, these quantities are roughly 30 orders of magnitudes apart.

We have also reduced the perturbation amplitude to $\epsilon=0.1$ in order to estimate the sensitivity of the results to this parameter. As we will see in Sect. 4, the onset of the magnetorotational instability is not affected by the smaller amplitude. The decay of differential rotation takes slightly longer with a factor of 1.6 but does not increase by an order of magnitude.

Are stars in the efficient regime of the magneto-rotational instability? For accretion disks rotating according to the Keplerian law where $\Omega \sim s^{-3 / 2}$, the wavelength of the most unstable mode is

$\lambda_{\text {inst }}=2 \pi \sqrt{16 / 15} u_{\mathrm{A}} / \Omega$

where $u_{\mathrm{A}}=B / \sqrt{\mu \rho}$ is the Alfvén velocity (Balbus \& Hawley 1998). Let us assume that the rotation profile is partly Keplerian in a star and the rotation period is 1 day. A magnetic field of $100 \mathrm{kG}$ will result - for densities between 1 and $10^{2} \mathrm{~g} / \mathrm{cm}^{3}-$ in wavelengths of 90000 to $9000 \mathrm{~km}$.

At this point, we can see that the magneto-rotational instability will set in much less promptly if $\lambda_{\text {inst }}>R$, thus imposing an upper limit to the magnetic field. In principle, Eq. (10) reflects the aforementioned fact that the magnetic energy must be significantly smaller than the kinetic energy in order to be called a "weak field". For stars, this is no problem as $\lambda_{\text {inst }}$ and $B$ are proportional, and the limiting magnetic fields for which $\lambda_{\text {inst }}>R$ are at least an order of magnitude larger than the fields assumed in the stellar interior.

There is no lower limit for $B$ in ideal MHD, but the nonvanishing diffusivity $\eta$ leads to a minimum magnetic field necessary for the magneto-rotational instability. Essentially, the growth rate of a perturbation with $\lambda_{\text {inst }}$ must be larger than the decay rate of a structure of the same wavelength. The former is independent of $\lambda$, whereas the latter changes with $\lambda^{-2}$. Balbus $\&$ Hawley (1998) give estimates of the minimum field for the limits of $P m \gg 1$ and $P m \ll 1$. Both cases lead to $B \approx 1 \mathrm{G}$ in stellar interiors, assuming $\eta=1000 \mathrm{~cm}^{2} \mathrm{~s}^{-1}$.

Even though magnetic fields in Ap stars are well in the suitable range for instability, we have to check the applicability of our initial $B$ for the numerical model, which requires a much larger magnetic diffusion. This strong diffusion makes the instability window much narrower than it is in reality.

Using the angular velocity of about 6000 at $s=0.6$, the lower, diffusive limit is $B=75$, while the upper constraint from the size $R$ of the domain is $B=920$. In the computational setup with $R m=10000$ and $B_{0}=100$, we obtain a wavelength of the most unstable mode of $\lambda_{\text {inst }}=0.1$ near $s=0.6$. Under real conditions, the range of suitable fields spans many orders of magnitudes, since $R$ is huge and $\eta$ is extremely small.

The values of $P m, R m$, the initial magnetic field strength $B_{0}$ and the amplitude of the perturbation, $\epsilon$, are the free parameters in the equations. Stellar gases possess magnetic Prandtl numbers of $P m \approx 0.01$. Values different from unity are typically difficult to achieve by numerical schemes. Values vastly different from unity mean that the time-scales for the diffusive processes in velocity and magnetic fields differ very much and are thus hard to cover appropriately by one simulation. We will vary $P m$ in our MHD simulations to evaluate the reasonableness of using $P m$ near unity.

The velocity and magnetic field in our computational domain are decomposed into 50 Chebyshev polynomials, 100 Legendre polynomials, and 30 Fourier modes. This resolution is sufficient to resolve the above wavelength of $\lambda_{\text {inst }}=0.1$ in our numerical setup. The nonlinear terms $-(\boldsymbol{u} \cdot \nabla) \boldsymbol{u}+(\nabla \times$ $\boldsymbol{B}) \times \boldsymbol{B}$ and $\nabla \times(\boldsymbol{u} \times \boldsymbol{B})$ are computed on a suitable number of collocation points in real space, and the spectral decomposition of these "right sides" are fed into the implicit timestepping scheme of the linear part of Eqs. (1) and (2). Apart from our standard resolution $(K, L, M)=(50,100,30)$, first simulations with lower resolutions of $(K, L, M)=(25,30,30)$ and $(K, L, M)=(50,50,30)$ led to the same results within $3 \%$.

The boundary condition for the flow is stress-free at the innermost and outermost radius. Vacuum conditions are imposed to the magnetic field at the inner and outer boundaries.

\section{Hydrodynamic evolution}

Before studying the magnetohydrodynamic case, we have to assess the evolution of the rotation flow without magnetic fields. This is of particular interest since the stress-free boundary conditions are not compatible with the initial azimuthal velocity profile $u_{\phi}(r \sin \theta)$. The rotation profile will lead to meridional circulations which equalize the differential rotation on the viscous time-scale. We thus need to determine the purely hydrodynamic decay time before we can turn to magnetic configurations and their instabilities.

We measure a decay time with $\Omega(r, \phi)$ in the equatorial plane. The quantity is averaged over $\phi$, providing a onedimensional function of $r$. The equatorial plane was chosen just for simplicity. Function (6) is fitted to that profile varying $R m$ and $q$. We determine the time when $q(t)=1$ and call it the decay time $\tau_{\text {decay }}$.

A model with $R m=10000$ gradually decays and reaches $q=1$ after 65 rotation periods $\tau_{\text {rot }}$. We will see later that this time is much longer than the decay time caused by the magneto-rotational instability. The kinetic energy in the meridional circulation is less than $1 / 2000$ of the energy of the azimuthal velocities. At $R m=50000$ the viscous decay lasts much longer than 200 rotation periods (Fig. 1). The ratio of azimuthal to meridional kinetic energies now exceeds $10^{4}$. Viscosities as low as stellar microscopic values are of course not achievable by numerical simulations. We can turn to magnetohydrodynamic computations knowing that the desired mechanism of differential-rotation decay should work on a time-scale of order 10 rotational periods or less to be essentially unaffected by viscosity.

\section{MHD evolution}

\subsection{Simulation results}

The following simulations including magnetic fields regard the enhanced decay rate of differential rotation as expected from the magneto-rotational instability. An initial magnetic field as 


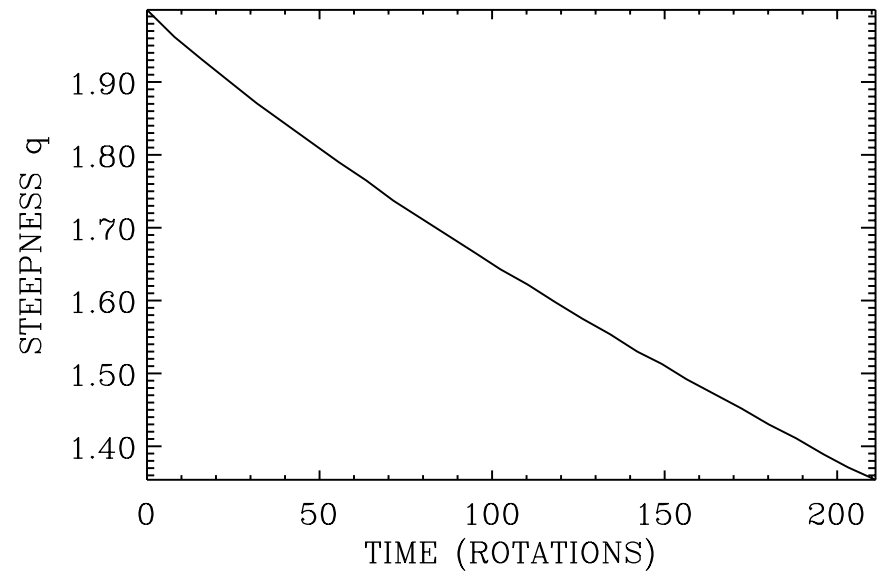

Fig. 1. Hydrodynamical computation of the viscous decay of differential rotation. A Reynolds number of $R m=50000$ was used here.

described in Eq. (9) in Sect. 2 causes poloidal flows with vortices of the same size as the perturbation of the magnetic field. They form in all places, except where the gradient of the angular velocity is negligible, i.e. close to the rotation axis. A series of vertical cuts through the velocity field at four equidistant times is shown in Fig. 2. The projected velocity vectors indicate that the problem is numerically resolved. Note that only every second vector of our collocation point grid is plotted for the sake of visibility; at radii smaller than 0.45 only every third vector. The same series is shown for the magnetic field in Fig. 3. The vector lengths in both graphs are not comparable among the four slices; they are scaled for best visibility.

The first velocity snapshot after 1.6 orbital revolutions shows the development of cells as the direct consequence of the Lorentz forces resulting from the magnetic-field perturbation. Two counter-rotating vortices represent one wavelength of the perturbation from the second term in (9). Roughly four of these waves fit into the sphere according to the wavenumber $k=4 \pi$. The second slice shows the emergence of relatively thin sheets of strong radial flows in up and down directions. These features become very prominent in the third figure after $4.8 \tau_{\text {rot }}$. They are actually quite extended over several tens of degrees in azimuthal direction. A detailed plot with the full resolution of our collocation point grid is shown in Fig. 4 magnifying a localized upstream. The fourth velocity slice of Fig. 2 shows an almost equalized rotation profile and a decay of small-scale features in the flow.

The latitudinal resolution of the model shown in Fig. 3 is plotted in Fig. 5 as a series of three Legendre spectra at $t=$ $0.001,0.003$, and 0.010 , corresponding to $1.6 \tau_{\text {rot }}, 4.8 \tau_{\text {rot }}$, and $16 \tau_{\text {rot }}$ resp. Maximum and minimum power span 2.5 orders of magnitudes in the most turbulent case (solid line). The initial power contrast is $10^{7}$ (dotted line); after the redistribution of angular momentum, the contrast quickly reaches the same order of magnitude again (dashed line in Fig. 5). The spectra of the velocity fields are very similar. The Fourier spectra as given in Fig. 6 show very satisfying power contrast all through the simulation.

The change of specific angular momentum as a function of axis distance is plotted in Fig. 7. The profiles of $s^{2} \Omega$ are - for simplicity - again taken from the equatorial plane and averaged over the $\phi$-direction. The initial differential rotation profile of (6) with $R m=10000$ and $q=2$ is shown as a flat curve, while the steepest, nearly parabolic lines are the final distribution of angular momentum, corresponding to a nearly uniform rotation. We plotted dashed lines for $t \geq 4.8 \tau_{\text {rot }}$; a gap between these and the solid lines marks the transition period when strongest transport of angular momentum is found.

The redistribution of angular momentum is a combined result of stresses from velocity and magnetic field fluctuations. The averages $\left\langle u_{\mathrm{r}}^{\prime} u_{\phi}^{\prime}\right\rangle$ and $-\left\langle B_{\mathrm{r}}^{\prime} B_{\phi}^{\prime}\right\rangle$, which are again taken in the equatorial plane only, show a clear domination of magnetic stresses over kinetic stresses, occasionally by a factor 10 .

In the same way as in Sect. 3 we measure the decay time of the differential rotation by the time it takes the system to cross a $q=1$ profile. Despite the enormous flows emerging, the azimuthally averaged angular velocity provides us with profiles $\Omega(r)$ in the equatorial plane which are fairly compatible with the two-parameter function (6). Figure 8 shows examples of such fits for two velocity snapshots of the simulation illustrated by snapshots in Figs. 2 and 3. It is thus still reasonable to use the steepness $q$ for defining the decay time even for the simulations where the magneto-rotational instability emerges.

The decay of $q$ with time is given by the solid line in Fig. 9. A short transition period between $4.0 \tau_{\text {rot }}$ and $5.5 \tau_{\text {rot }}$ can be seen. The third velocity slice of Fig. 2 with strong radial up and down flow sheets falls right in the middle of this period. Towards the end of the computation, the system oscillates around an equilibrium state with $q=0$, and magnetic and kinetic energies decay exponentially.

The transition period is less marked when we go to lower diffusivities, i.e. to higher magnetic Reynolds numbers $R m$. The steepness $q$ diminishes more gradually, but still on a timescale which is an order of magnitude shorter than viscous decay in purely hydrodynamic simulations. (Note, however, that the difference to viscous decay is expected to be much larger in a real star.) We have added the non-magnetic model with $R m=10000$ as a dashed line in Fig. 9 illustrating the marginal influence of viscous decay on the MHD simulations.

\subsection{Application to stellar parameters}

The rotation profile seems to decay on the rotational time-scale. This is apparently far too fast for any trace of $\Omega(s)$-rotation in stars with radiative envelopes. We will later see that this is not quite true. There are the real physical quantities which are hard to match in a computer simulation. The diffusive time-scale is orders of magnitudes longer than the rotational time-scale. We managed to make them more than four orders of magnitudes different and may obtain an extrapolation towards real stellar parameters. The magnetic Reynolds number $R m=R^{2} \Omega / \eta$ in stellar radiative zones is about $10^{13}-10^{14}$. The highest $R m$ achieved numerically is 50000 in this presentation. The dependence of the decay time $\tau_{\text {decay }}$ on the magnetic Reynolds number and magnetic Prandtl number is shown in Fig. 11. The decay times are given in rotation periods, which is a few days for CP stars. Fortunately, we found no significant dependence 

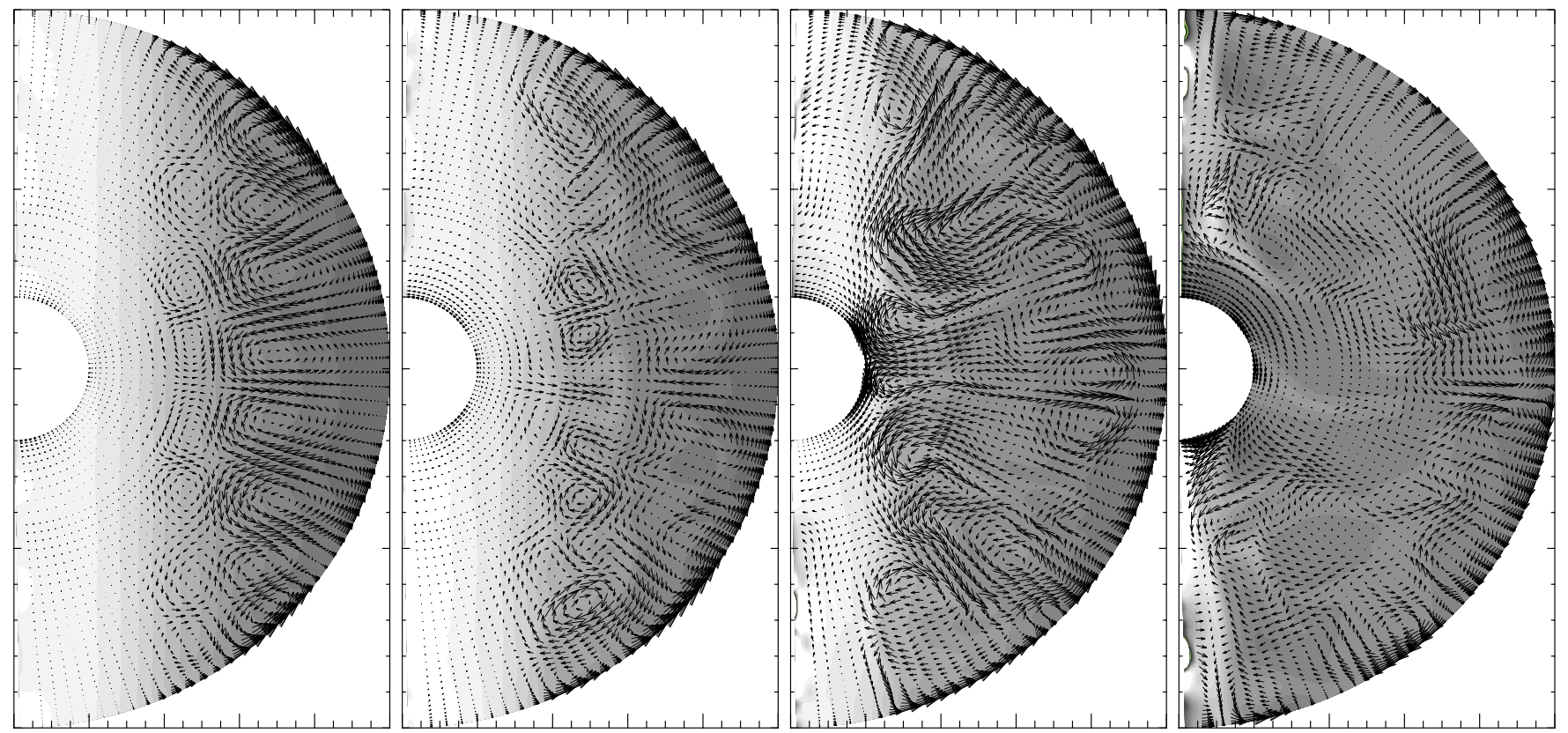

Fig. 2. Series of velocity snapshots for the model with $R m=10000$ and $P m=1$ at $t=0.001,0.002,0.003,0.004$ diffusion times (1.6, 3.2, 4.8 , and $6.4 \tau_{\text {rot }}$ ). The grey level contours refer to the angular velocity $u_{\phi} / s$. The scaling of these contours is the same in all four cross-sections, while the scaling of the vector lengths varies. For better visibility, we plotted only every second vector; only every third below $r=0.45$.
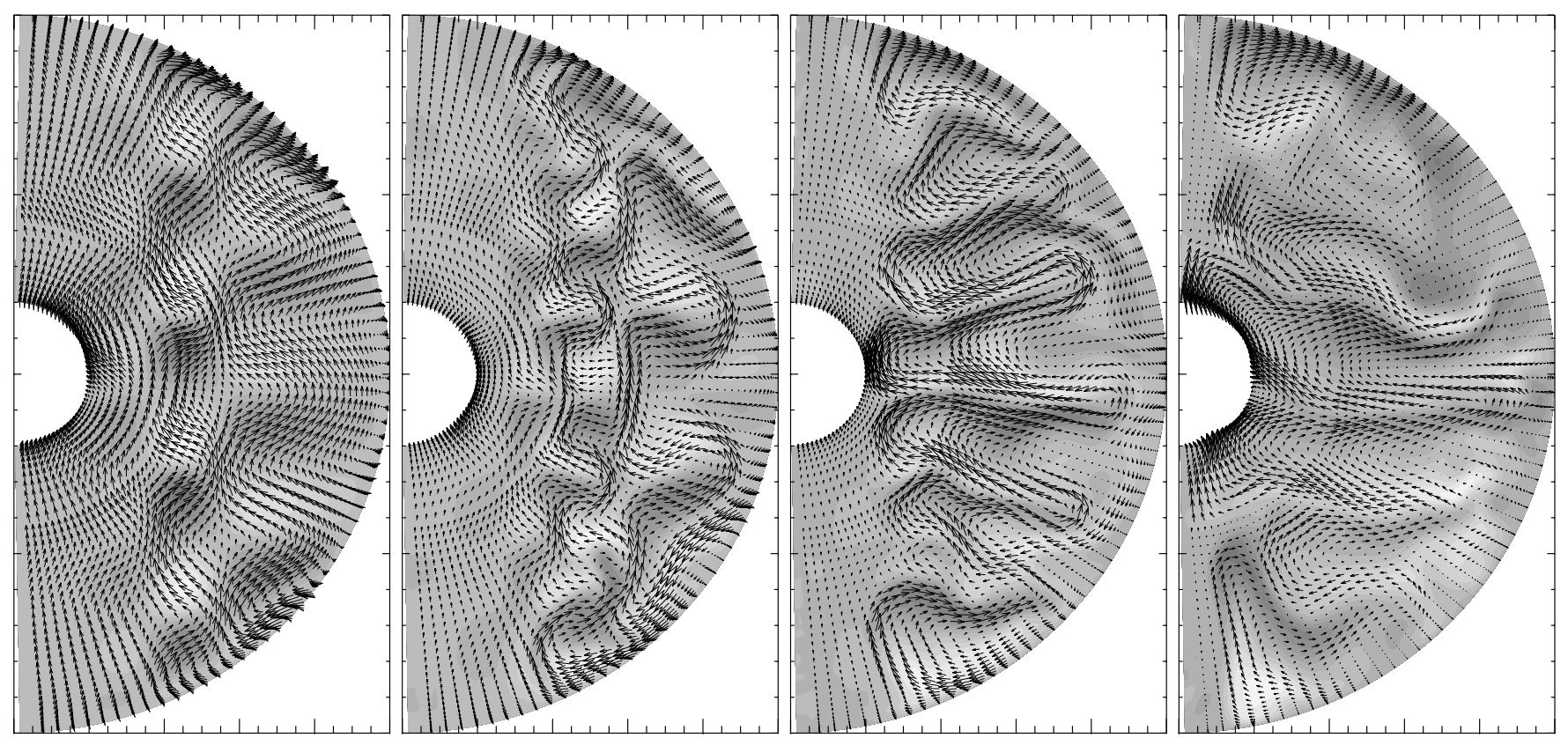

Fig. 3. Series of snapshots of the magnetic field for the model with $R m=10000$ and $P m=1$ at $t=0.001,0.002,0.003,0.004$ diffusion times $\left(1.6,3.2,4.8\right.$, and $\left.6.4 \tau_{\text {rot }}\right)$. The grey level contours refer to $B_{\phi}$. Again we plotted only every second vector; only every third below $r=0.45$.

of the decay times on $\mathrm{Pm}$. As the true magnetic Prandtl number will be of the order of $10^{-2}$ for stars, we may assume that our $P m$ near unity will not imply severe differences from the real physics.

Also the amplitude $\epsilon$ of the perturbation of the initial vertical magnetic field was changed to $\epsilon=0.1$. The resulting decay of differential rotation for the model with $R m=10000$ and $P m=1$ is shown in Fig. 10 in terms of the steepness $q$ of the rotation profile. The decay time increased from 5 to 8 rotation periods. Also the ratio of magnetic to kinetic stresses as the constituents of the transport of angular momentum is not changed and reaches 10:1.

With a series of computations with various $R m$, we can make an attempt to extrapolate the decay time to stellar conditions. It is found that the decay time scales as the magnetic Reynolds number, in particular for $R m \geq 10000$ which is the interesting interval. Measured in diffusion times, the decay time depends only slightly on $R m$. We derive the relation

$\frac{\tau_{\text {decay }}}{\tau_{\text {rot }}}=\frac{R m}{2000}$ 


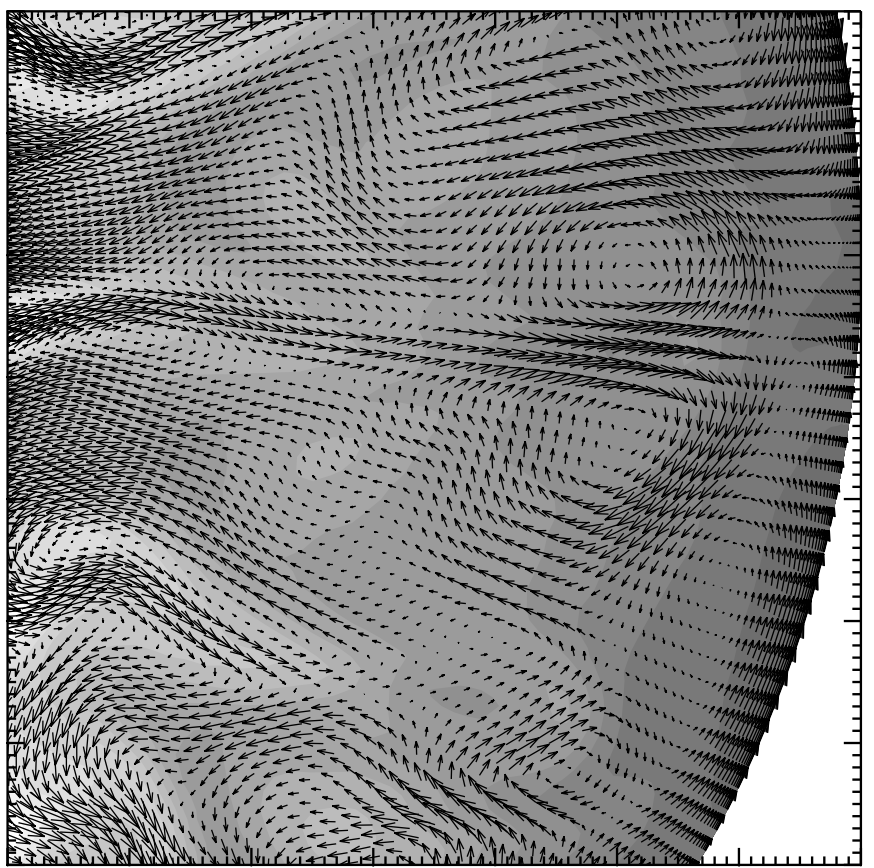

Fig. 4. Detail of the velocity field shown in the third slice in Fig. 2 with full collocation point resolution.

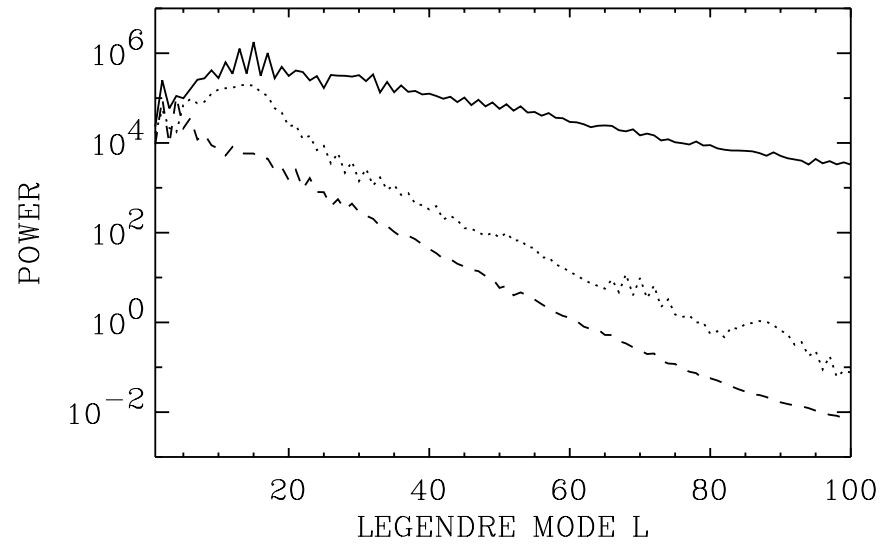

Fig. 5. Legendre spectra (latitudinal decomposition) of the energy of the magnetic field for the model with $R m=10000$ and $P m=1$ at three instances. Dotted line: $t=0.001$ in the beginning of the instability; solid line: $t=0.003$ during the period of strongest flows (transition period); and the dashed line: $t=0.010$ when velocity and magnetic fields decay. These times correspond to 1.6, 4.8, and $16 \tau_{\text {rot }}$.

where $\tau_{\text {rot }}$ is the rotation period of the star. If the stellar parameters $R=2 R_{\odot}, \tau_{\text {rot }}=1$ day, and $\eta=1000 \mathrm{~cm}^{2} \mathrm{~s}^{-1}$ (Spitzer 1956) are applied, the magnetic Reynolds number is $3 \times 10^{14}$. The diffusivity is the variable ingredient here; our diagram in Fig. 11 can thus be annotated with $\eta$ instead of $R m$ (see upper abscissa). If $R m$ enters (11) with an exponent unity, the decay time is actually locked to the diffusion time and not to the rotation period. If we adopt a stellar $P m=10^{-2}$ and rewrite Eq. (11) as $\tau_{\text {decay }} / \tau_{\text {rot }}=0.0005 P m R e$, we see that the differential-rotation decay as a consequence of the magnetorotational instability is of $O\left(10^{5}\right)$ times faster than the viscous decay scaling with $R e$.

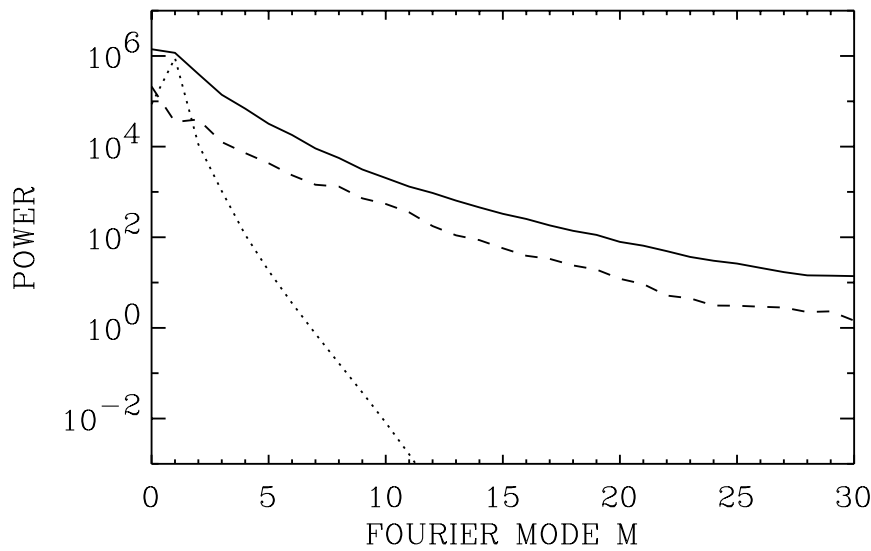

Fig. 6. Azimuthal decomposition in terms of Fourier spectra of the magnetic field energy for the model with $R m=10000$ and $P m=1$ at the same times $t=0.001$ (dotted), 0.003 (solid), and 0.010 (dashed) as in Fig. 5.

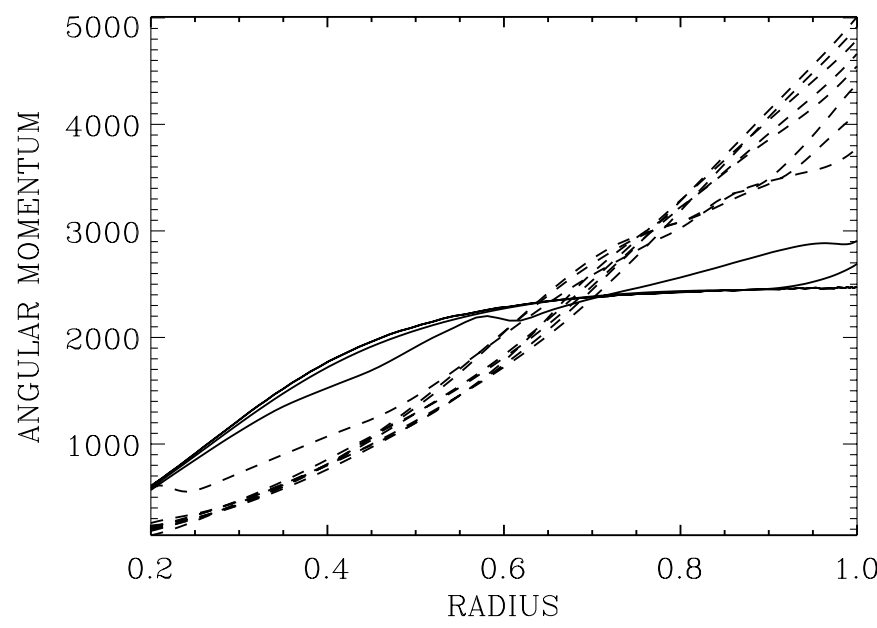

Fig. 7. Redistribution of angular momentum $j$ in the model with $R m=10000$ and $P m=1$. The same equatorial averages as applied for the determination of the steepness $q$ were used, and $j$ was plotted for various times of the simulation in steps of $1.6 \tau_{\text {rot }}$; dashed lines are for $t \geq 4.8 \tau_{\text {rot }}$. The flat profile is from the initial rotation profile, the steep, nearly parabolic profile is the final state with almost constant angular velocity.

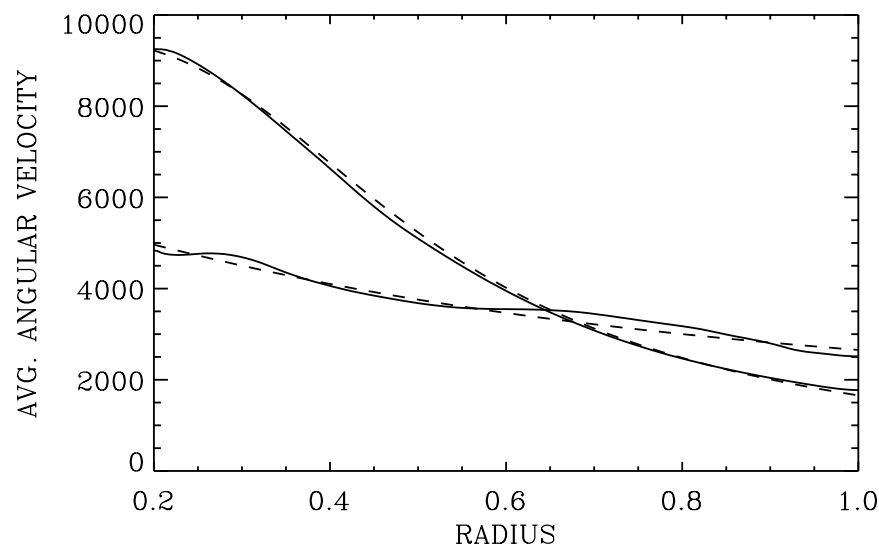

Fig. 8. Two examples of the angular velocity averaged in the equatorial plane as a function of radius (solid lines) and the fit of (6) with $R m$ and $q$ (dashed lines) at $t=0.0015\left(2.4 \tau_{\text {rot }}\right)$ and $t=0.0032\left(5.1 \tau_{\text {rot }}\right)$. 


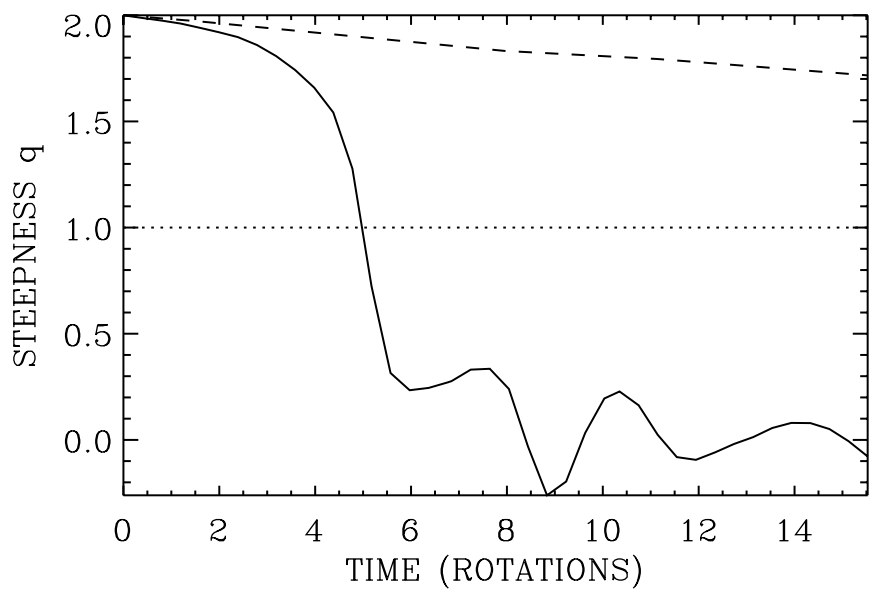

Fig. 9. Variation of the steepness of the rotation profile with time as computed from azimuthal averages of the angular velocity of the model with $R m=10000, P m=1, B_{0}=100$, and $\epsilon=1$. The resulting function of $r$ was fitted to a function of the form (6). The dotted line indicates $q=1$ which will be used to define the decay time of the differential rotation. The dashed line shows the change of $q$ in a hydrodynamic model where the differential rotation decays only by viscosity.

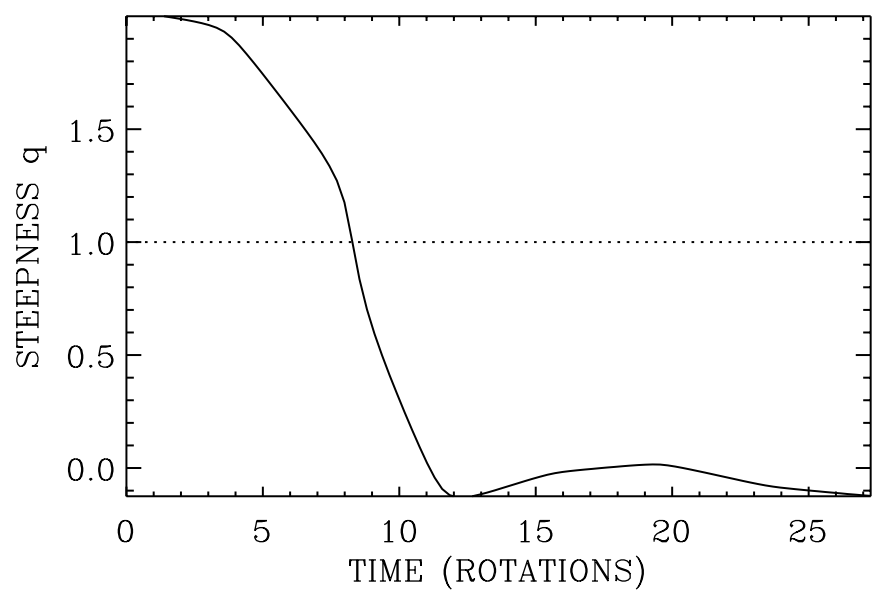

Fig. 10. Variation of differential rotation of the model with $R m=$ 10000 as in Fig. 9. The initial perturbation of the vertical magnetic field, however, was a factor 10 smaller than there, $\epsilon=0.1$.

Relation (11) delivers a decay time of $3 \times 10^{8} \mathrm{yr}$ for the stellar parameters given above. This is of the order of the lifetime of an A star. Of course, this extrapolation involves considerable uncertainty. If we look at the graph for $P m=10$, the power of $R m$ is slightly lower, $\tau_{\text {decay }} / \tau_{\text {rot }}=0.002 R m^{0.85}$. The resulting decay time for $R m=3 \times 10^{14}$ is $\tau_{\text {decay }}=10^{7} \mathrm{yr}$.

\section{Density stratification}

The results of Sects. 3 and 4 are limited to a homogeneous fluid sphere, and we would like to address the influence of a density stratification here. The numerical scheme can be extended to a background density stratification which does not vary with time. The quantities $e$ and $f$ of Eq. (4) now constitute the mass flux rather than the velocity. Additional diffusive terms and nonlinear terms were implemented, and boundary conditions

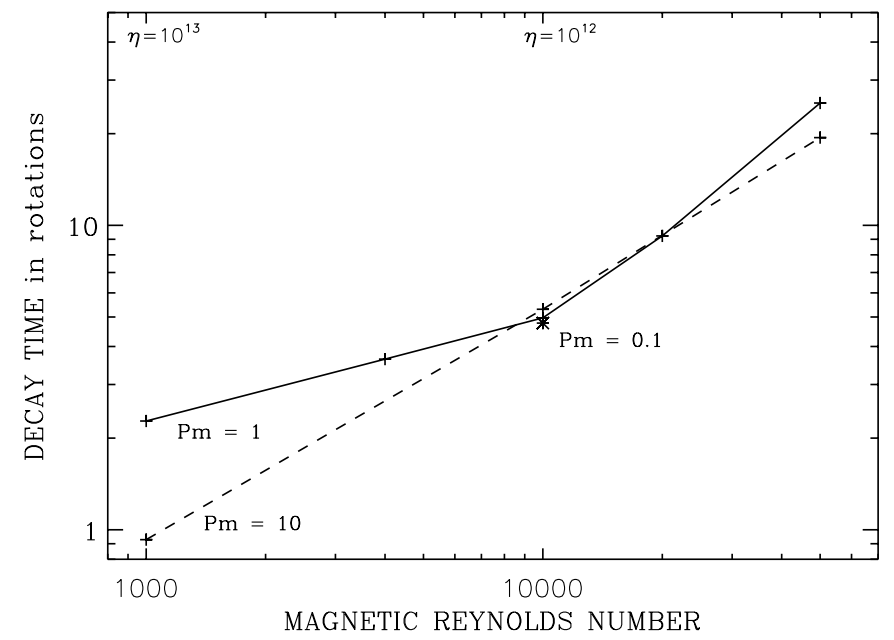

Fig. 11. Decay time of differential rotation versus magnetic Reynolds number for nine simulations with high spectral resolution. The decay time is measured in rotations. The upper abscissa gives an idea of the magnetic diffusivities $\eta$ implied by the Reynolds numbers, assuming a stellar radius and stellar rotation rate. The solid line refers to $P m=1$, the dashed line shows the results of $P m=10$. An asterisk indicates the decay time for the only computation with $P m=0.1$.

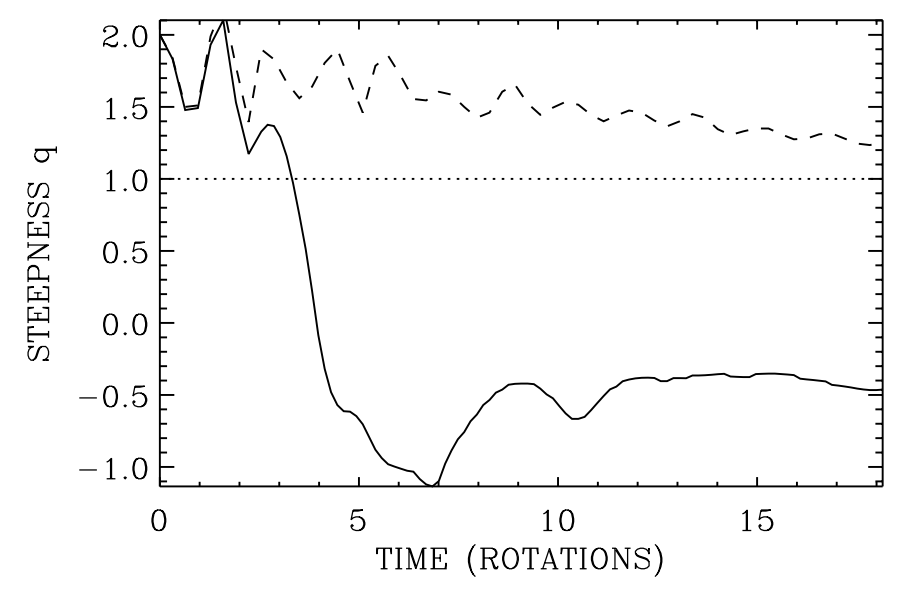

Fig. 12. Development of the steepness $q$ of the rotation profile for a model with background density stratification. The dashed line is the hydrodynamic evolution, the solid line is the evolution with magnetic fields.

adapted to retain stress-free surfaces. The density stratification is given by

$\rho(r)=\rho_{0} \exp \left(-r / r_{0}\right)$

where $\rho_{0}$ is the central density and $r_{0}$ is the scale-height; here $r_{0}=0.8$, giving a density contrast of $2.7: 1$ between the inner and outer boundaries. The initial rotation profile and magnetic field are the same as in Eqs. (6) and (9) using the parameters for Fig. 9.

The hydrodynamic and MHD evolutions of this configuration are shown in Fig. 12. Since a profile $\Omega(s)$ is no longer compatible with the Taylor-Proudman theorem, circulations lead to stronger variations of $q$ even in the hydrodynamic case. Yet, the actual decay, as seen from the dashed line, is still much slower 


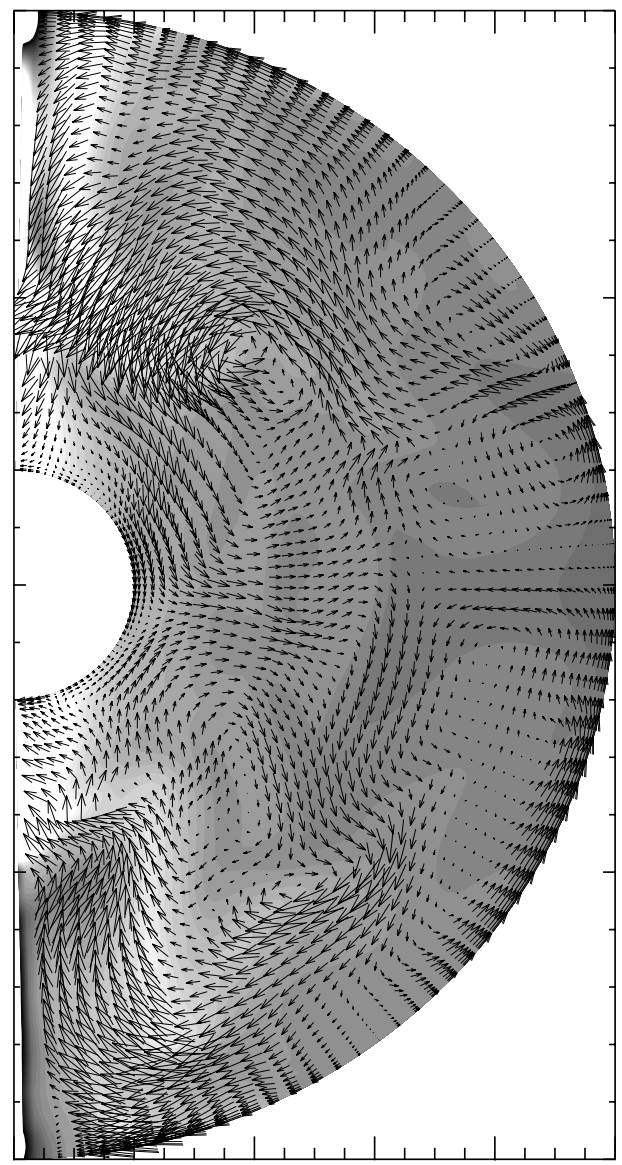

Fig. 13. Velocity snapshot for a model with radial background density stratification. The slice was taken at $t=0.002$ (corresponding to $3.2 \tau_{\mathrm{rot}}$ ).

than the MHD decay shown as a solid line. The MHD decay has reduced from $5 \tau_{\text {rot }}$ to $3.5 \tau_{\text {rot }}$.

The meridional cut through the shell in Fig. 13 shows the projected velocity vectors and the grey-coded angular velocity after $3.2 \tau_{\text {rot }}$ and represents the flow shortly before reaching $q=1$. Fewer straight up- or downflows are visible than in Fig. 2. Strong flows are constrained to high stellar latitudes. It should also be noted that the flow over-compensates the original differential rotation and leads to a weak, positive gradient in $\Omega(s)$ at the equator. This gradient appears to decay on the viscous time-scale. Nevertheless, this gradient is smaller than the negative gradient remaining from purely hydrodynamic simulations after the same time.

\section{Summary}

Since differential rotation in radiative stellar zones cannot be damped by viscosity within the life-time of a star, we investigate the magnetic evolution that is likely to imply the onset of the magneto-rotational instability providing efficient angular-momentum transport. MHD simulations of spherical shells were performed showing that the instability does indeed emerge, and rapid equalization of differential rotation is found. An extrapolation to stellar parameters gives decay times of differential rotation of the order of 10-100 million years. This is the time-scale over which redistribution of angular momentum is taking place. The magneto-rotational instability grows on a scale of rotation periods, but the net efficiency of angular momentum transport in the fully nonlinear regime is obviously a different one. First tests with stratified spherical shells indicate similar or even higher efficiencies in the redistribution of angular momentum, although the flow patterns are different from the patterns in a non-stratified configuration. We would like to address the effects of buoyancy and its combination with density stratification in a future contribution. Since magnetic forces act in a way to reduce their origin, we should not forget that we can expect the magnetic-field perturbations to damp differential rotation in any case. The key question here is the timescale on which this happens. Buoyancy is supposed to suppress radial angular-momentum transport (Balbus \& Hawley 1994), and an increase in decay times is thus likely to occur in a refined model.

Bearing in mind that the decay times obtained here are possibly lower limits, we may conclude the following items: while solar-type stars have sufficiently long life-times to rotate uniformly in their radiative cores, the life-time of stars of spectral type $A$ is of the same order of magnitude as the decay time of about 100 million years. CP stars have magnetic fields, providing the conditions for the magneto-rotational instability - along with an initial differential rotation caused by interactions with the accretion disk of its pre-main sequence life. Because of the comparable stellar life-time and decay time, differential rotation should thus be present in CP stars during a considerable period of their life. As long as the total angular momentum is conserved, we should expect a slight increase of surface angular momentum with age. Since an A star roughly doubles its radius during its presence on the main sequence, the resulting decrease of surface rotation may balance the increase due to the mechanism described in this paper. Age data and precise rotation periods of $\mathrm{CP}$ stars will be needed to test this result.

\section{References}

Arlt, R., \& Rüdiger, G. 2001, A\&A, 374, 1035

Balbus, S. A., \& Hawley, J. F. 1991, ApJ, 376, 214

Balbus, S. A., \& Hawley, J. F. 1994, MNRAS, 266, 769

Balbus, S. A., \& Hawley, J. F. 1998, Rev. Mod. Phys., 70, 1

Bouvier, J., Cabrit, S., Fernandez, M., Martin, E. L., \& Matthews, J. M. 1993, A\&A, 272, 176

Cameron, A. C., \& Campbell, C. G. 1993, A\&A, 274, 309

Hawley, J. F. 2000, ApJ, 528, 462

Hollerbach, R. 2000, Int. J. Numer. Meth. Fluids, 32, 773

Kitchatinov, L. L., \& Rüdiger, G. 1997, MNRAS, 286, 757

Landstreet, J. D., \& Mathys, G. 2000, A\&A, 359, 213

Spitzer, L. Jr. 1956, Physics of fully ionized gases (New York Interscience Publ.), 81

Stępień, K. 2000, A\&A, 353, 227 